\title{
Técnica EXIT como manejo de la vía aérea en masas gigantes congénitas de cuello
}

\author{
Ex-Utero Intrapartum Treatment for airway management in congenital \\ giant neck masses
}

\author{
Jaime Orrego G. ${ }^{a}$, Juan Camilo Mosquera-Hernández $z^{\mathrm{b}}$, Santiago Ardila-Giraldo \\ Laura Torres-Canchala ${ }^{c}$, Edgar Alzate $^{\mathrm{d}}$, Juan Pablo Benavidez
}

\author{
aUnidad de Recién Nacidos, Fundación Valle del Lili, Cali, Colombia \\ bFacultad de Ciencias de la Salud, Universidad Icesi, Cali, Colombia \\ 'Centro de Investigaciones Clínicas, Fundación Valle del Lili, Cali, Colombia \\ ¿Servicio de Cirugía Pediátrica, Fundación Valle del Lili, Cali, Colombia \\ eUnidad de Alta Complejidad Obstétrica, Fundación Valle del Lili, Cali, Colombia
}

Recibido: 19 de agosto de 2019; Aceptado: 17 de noviembre de 2019

¿Qué se sabe del tema que trata este estudio?

La técnica EXIT ha sido confirmada como un abordaje seguro y eficaz en condiciones congénitas con riesgo de asfixia perinatal por compresión de la vía área que disminuye morbimortalidad. Para su ejecución se requiere del diagnóstico oportuno y de un equipo multidisciplinario.
¿Qué aporta este estudio a lo ya conocido?

Esta investigacion contribuye a la estandarización de esta técnica en países de medianos ingresos.

\section{Resumen}

Las masas congénitas de cabeza y cuello se asocian a asfixia perinatal e injuria cerebral con elevada mortalidad. La técnica EXIT (Ex Útero Intrapartum Treatment) consiste en asegurar la vía aérea del neonato, sin interrumpir la oxigenación y perfusión materno-fetal a través del soporte placentario. Esta técnica no ha sido estandarizada en países de medianos ingresos. Objetivo: Describir el caso clínico de 2 neonatos manejados mediante la técnica EXIT. Caso Clínico: Se reportan dos casos, uno con malformación linfática diagnosticada a la semana 20 gestación y el segundo con tiromegalia y polihidramnios diagnosticados a la semana 35 de gestación. En ambos casos, durante la cesárea se realizó la técnica EXIT con un equipo conformado por neonatólogo, ginecólogo, anestesiólogo, cirujano pediatra, otorrinolaringólogo, enfermero y terapeuta respiratorio. En los dos pacientes se logró asegurar la vía aérea mediante intubación orotraqueal al primer intento. En
Palabras clave:

Intubación

Orotraqueal;

Recién nacido;

Neoplasias de cabeza y cuello;

Ex Utero Intrapartum

Treatment;

EXIT 
el caso 1 se confirmó la malformación linfática y recibió escleroterapia, y en el caso 2 se diagnosticó hipotiroidismo congénito asociado a bocio, que fue manejado con levotiroxina. Los pacientes se mantuvieron 7 y 9 días con ventilación mecánica invasiva respectivamente y egresaron sin complicaciones respiratorias. Conclusiones: La técnica EXIT en estos casos fue un procedimiento seguro, llevado a cabo sin inconvenientes. Se necesita un equipo multidisciplinario y la disponibilidad de una unidad de cuidados intensivos neonatales, con el objetivo de reducir potenciales complicaciones y garantizar el manejo postnatal. Para lograr su ejecución, es indispensable el diagnóstico prenatal oportuno.

\section{Keywords:}

Orotracheal Intubation; Newborn;

Head and Neck

Neoplasms;

Ex Utero Intrapartum

Treatment;

EXIT

\section{Introducción}

Las masas congénitas de cabeza y cuello como parte del síndrome de obstrucción congénita de las vías respiratorias altas (CHAOS en inglés) se asocian con alta mortalidad e injuria cerebral hipóxico-isquémica en recién nacidos $(\mathrm{RN})^{1,2}$. Las causas más frecuentes son las malformaciones linfáticas, que ocurren en $1 / 1775$ nacidos vivos ${ }^{3}$. Las masas congénitas de cabeza y cuello pueden provocar una compresión y distorsión grave de la vía aérea lo que genera alta dificultad de asegurar el soporte ventilatorio al momento del nacimiento, aumentando el riesgo de muerte o de complicaciones por hipoxia perinatal. Con la finalidad de prevenir estos desenlaces, la técnica EXIT (Ex Utero Intrapartum Treatment) se ha catalogado como una manera segura de intervenir la vía aérea del paciente evitando la alteración de la perfusión de oxígeno tisular del RN. Para esto se requiere la intervención de un equipo multidisciplinar de neonatólogos, otorrinolaringólogo, anestesiólogo, gineco-obstetra, cirujano pediatra, enfermeros y terapeutas respiratorios entrenados en cuidado materno-fetal. Por ello, el diagnóstico prenatal a través de una resonancia magnética (RM) es fundamental ${ }^{4}$.

La técnica EXIT, descrita por Norris et col en $1980^{5}$, consiste en asegurar a la vía aérea de manera controlada mediante intubación orotraqueal (IOT), fibrobroncoscopia rígida o traqueostomía (de acuerdo a la severidad de la obstrucción) sin interrumpir la circulación materno-fetal a través del soporte placentario el cuál ejerce las funciones respiratoria y cardiaca ${ }^{6-8}$. Una vez logrado esto se corta el cordón umbilical y se atiende a la madre y al RN por separado de acuerdo con sus requerimientos. Fue inicialmente recomendado para el manejo de la oclusión traqueal en $\mathrm{RN}$ con hernias diafragmáticas congénitas severas ${ }^{9,10}$ pero se extrapoló rápidamente a pacientes con CHAOS o con lesiones pulmonares congénitas ${ }^{11}$.

Aunque Cruz-Martínez et col en $2015^{12}$ describió la técnica EXIT en México, esta técnica no ha sido completamente estandarizada en países de medianos ingresos. El objetivo de este manuscrito es describir los desenlaces clínicos de dos pacientes en quienes se llevó a cabo este procedimiento y realizar una revisión del 
desarrollo de la técnica EXIT en un hospital de tercer nivel del suroccidente colombiano.

\section{Caso Clínico}

\section{Caso 1}

RN femenino, hija de primigestante de 21 años, con controles prenatales iniciados a las 9 semanas de gestación. A la semana 20 la ecografía detectó masa cervical de características quísticas. Posteriormente, una resonancia magnética (RM) evidenció gran masa que comprometía cuello y tórax derecho, extendiéndose entre el pabellón auricular y región torácica derecha, con un tamaño estimado de 12 x $15 \mathrm{~cm}$ (figura 1a). Se programó cesárea para la semana 36 de gestación, durante la cual se llevó a cabo la técnica EXIT y el neonatólogo logró asegurar la vía aérea con IOT en el primer intento (figura 1b), luego se trasladó a la unidad de cuidado intensivo neonatal.

La RM posnatal reportó hallazgos acordes con una malformación linfática. Se inició escleroterapia con adecuada respuesta clínica (figura 1c). Al día 7 de vida se retiró IOT, al día 16 se suspendió el soporte ventilatorio y se dio de alta al día 21 de vida sin complicaciones respiratorias.

\section{Caso 2}

RN masculino, segundo hijo de una madre de 17 años, que inició controles prenatales a la semana 30 gestación. Una ecografía a la semana 33 detectó polihidramnios leve. A la semana 35 se evidenció masa en cuello con marcada vascularización de tamaño estimado de $4 \times 3 \mathrm{~cm}$ en región anterior del cuello, desde esternón hasta el maxilar en lado izquierdo, que generaba compresión de la tráquea y esófago. A la semana 36 EG se indicó cesárea y durante la misma se realizó la técnica EXIT. El neonatólogo logró IOT al primer intento, y se trasladó a la unidad de cuidado intensivo neonatal para su cuidado posnatal.

La RM reportó una lesión de 5 x $3 \mathrm{~cm}$ que provocaba efecto de masa con contornos lobulados en el espacio mucoso faríngeo del cuello en la región anterior que rodea la tráquea, el esófago y desplazaba en sentido lateral y posterior los vasos carotideos y yugulares bilaterales sin comprimir la vía aérea (figura 2). Los niveles de THS se encontraron elevados con T3 y T4 disminuidos lo que confirmó el diagnóstico de hipotiroidismo congénito, que, asociado al importante aumento de volumen de la glándula, configura el diagnostico probable de dishormogénesis tiroidea. Se inició levotiroxina con adecuada respuesta clínica. Al 9 día de vida se retiró el tubo orotraqueal y continuó con ventilación mecánica no invasiva hasta el día 12 . Se dio de alta a los 14 días de vida sin complicaciones respiratorias, con manejo ambulatorio.
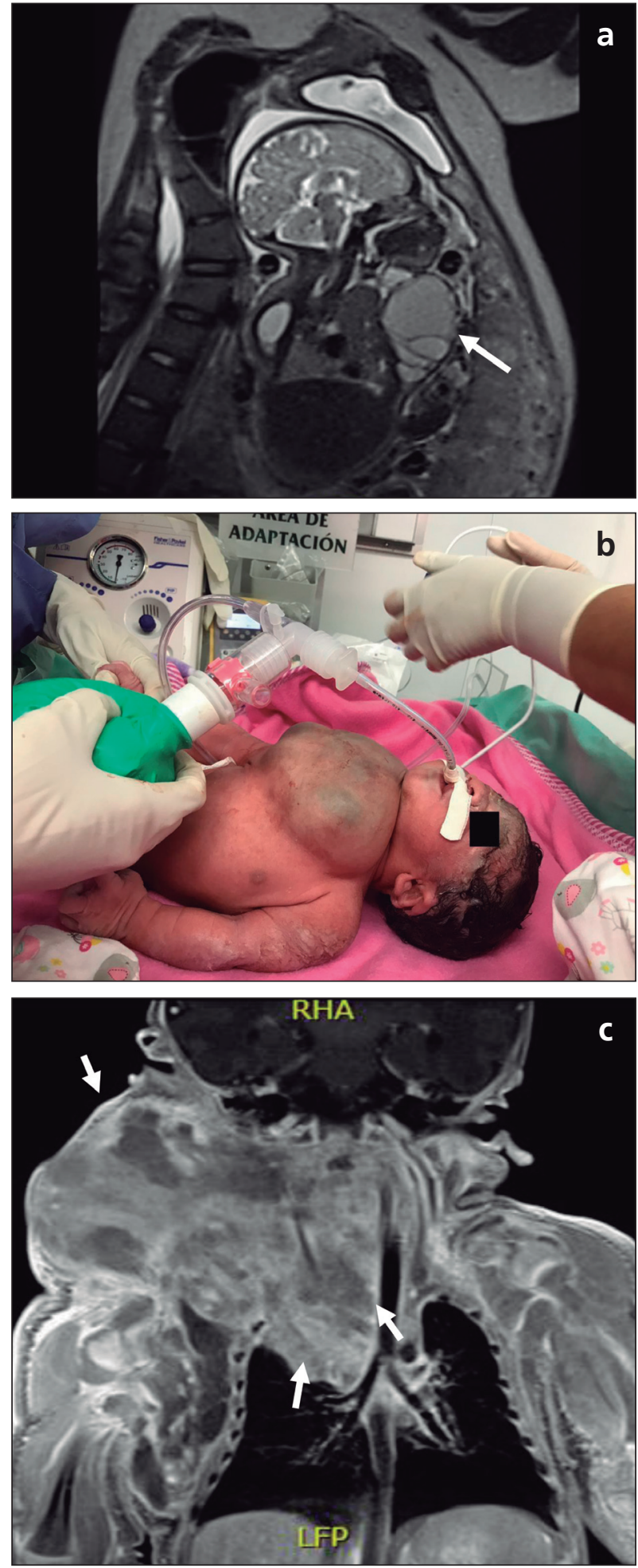

Figura 1. a) Resonancia Magnética (RM) fetal muestra gran masa multiquística que compromete hemicuello derecho, fosa supraclavicular, fosa axilar y pared posterolateral del tórax derecho compatible con malformación vascular veno-linfática (Flecha blanca), sin evidencia de compresión de la vía aérea; b) Fotografía de recién nacido femenino con malformación veno-linfática en cuello y tórax anterior izquierdo; c) RM posnatal muestra gran masa multiquística, con compromiso de espacio cervical posterior, carotideo y retrofaríngeo derecho, con extensión a al mediastino y a pared torácica anterior y posterior derecha (Flechas blancas) 


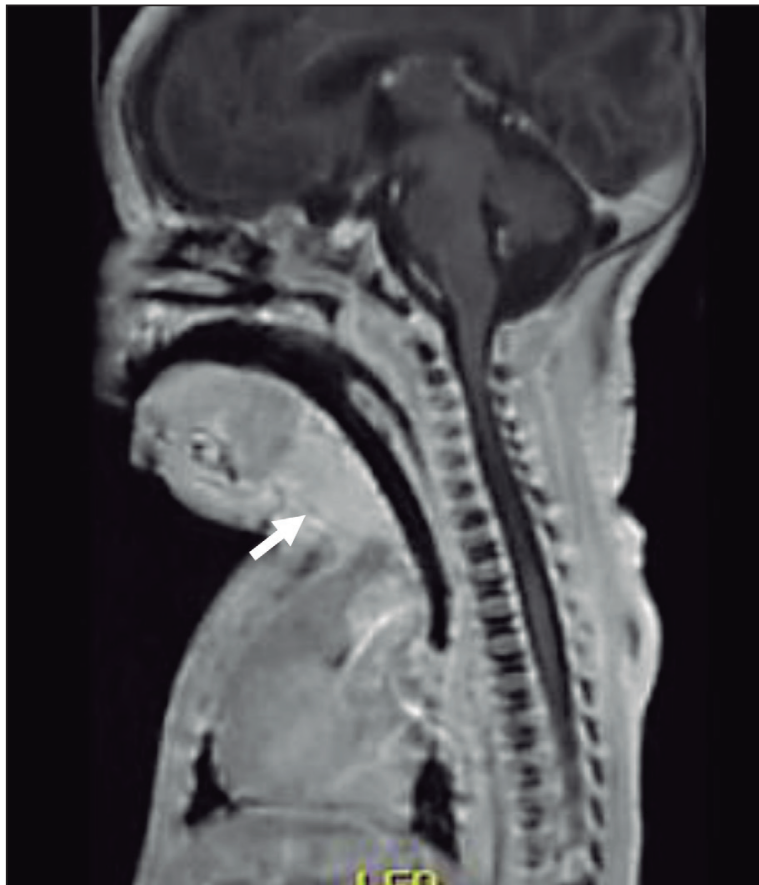

Figura 2. Resonancia Magnética postnatal corte sagital muestra importante tiromegalia /bocio.

\section{Discusión}

En los pacientes descritos la obstrucción de las vías respiratorias en los fetos generaba alto riesgo de hipoxia grave o la muerte. La implementación de la técnica EXIT en ellos fue la opción más segura y eficiente para mantener la perfusión tisular disminuyendo el riesgo de complicaciones a subyacentes a la asfixia perinatal.

La técnica EXIT fue inicialmente descrita en pacientes con oclusión traqueal pero rápidamente se ha extendido su uso incluyendo malformaciones pulmonares expansivas como malformaciones adenomatoides quísticas congénitas que puede comprimir el tejido pulmonar normal restante ${ }^{13}$, membrana laríngea, atresia o estenosis de la tráquea, anormalidades torácicas, entre otras que se muestran en la tabla $1^{14,15}$.

La técnica EXIT debe realizarse bajo anestesia general para asegurar la hipotonía del útero y permitir así la adecuada circulación placentaria, disminuir el riesgo de prolapso del cordón umbilical y de desprendimiento placentario ${ }^{16}$. Se requiere un equipo multidisciplinario entrenado y la disponibilidad de una unidad de cuidados intensivos neonatales ${ }^{8}$. Para ello, es necesaria la planeación del evento con base en las necesidades de cada paciente y por ende el diagnóstico temprano es fundamental para implementarla. En los casos descritos uno de ellos tuvo controles prenatales tardíos, condición que aumenta el riesgo de complicaciones generadas por su ejecución sin previa preparación. Las funciones de cada integrante del equipo participante se describen en la tabla 2.

El algoritmo de la técnica EXIT se describe en la figura 3. La incisión quirúrgica puede ser mediana abdominal o transversal inferior (tipo Pfannenstiel) y posterior a la histerotomía transversal inferior (tipo Keer), se extrae la cabeza fetal, la parte superior del tórax y al menos una extremidad superior ${ }^{4}$. Como es necesario evitar el esfuerzo respiratorio y los movimientos del cuerpo para lograr la intubación orotraqueal, puede ser necesario administrar anestesia fetal justo después de extraer un miembro superior, por vía intramuscular en el deltoides ${ }^{17,18}$. El resto del cuerpo permanece dentro de la cavidad uterina. Se recomienda infundir solución Hartman a $37^{\circ} \mathrm{C}$ en forma continua para conservar el volumen y la temperatura de la cavidad uterina. Puede estar indicado utilizar un oxímetro en la extremidad superior para monitorizar frecuencia cardiaca y saturación de $\mathrm{O}_{2}{ }^{12}$.

El soporte del oxígeno placentario al feto puede prolongarse, en óptimas condiciones, hasta $150 \mathrm{~min}^{19}$. La IOT es la medida principal de abordaje. En caso de falla, la IOT por broncoscopia y la traqueotomía, son la segunda y tercera opción de manejo ${ }^{20,21}$. En algunos casos será necesaria la resección parcial o completa de la masa para liberar la tráquea de la compresión externa que permita intubar o realizar traqueotomía y acceder

\section{Tabla 1. Indicaciones para implementación} de la técnica EXIT

- Retiro de clips en casos de Hernia Diafragmática Congénita $^{9}$

Garantizar una vía aérea permeable:

- Obstrucciones extrínsecas (teratomas, linfangiomas, bocio $)^{7,27}$

- Obstrucciones intrínsecas de la vía aérea (CHAOS, atresia laríngea o traqueal)

Extirpar masas intratorácicas

- Malformación adenomatosa quística

- Secuestro pulmonar

- Teratomas $^{28}$

Realizar la conexión a membranas de oxigenación extracorpórea (ECMO) en hernias diafragmáticas ${ }^{29}$

Separación de siameses

Otras indicaciones ${ }^{30}$ :

- Punción percutánea guiada por ecografía durante el procedimiento EXIT en linfangiomas gigantes cervicales

- Revertir oclusiones traqueales fetales en hernias diafragmáticas congénitas

- Anormalidades torácicas: derrames pleurales masivos y MAQ gigantes

- Eliminación de shunt toraco-amnióniticos. Toracotomías y resecciones de lóbulos pulmonares

- Canalización de vasos centrales previas al ECMO 
Tabla 2. Equipo interdisciplinar requerido para la técnica EXIT

\begin{tabular}{ll}
\hline Especialidad & Función \\
\hline Anestesiología & - Anestesia y relajación de la madre para mantener perfusión adecuada del feto \\
Ginecobstetricia & - Cesárea, extracción de cabeza y brazo derecho sin interrupción de circulación placentaria, que \\
permita el abordaje de la vía aérea fetal
\end{tabular}

Figura 3. Algoritmo de la implementación

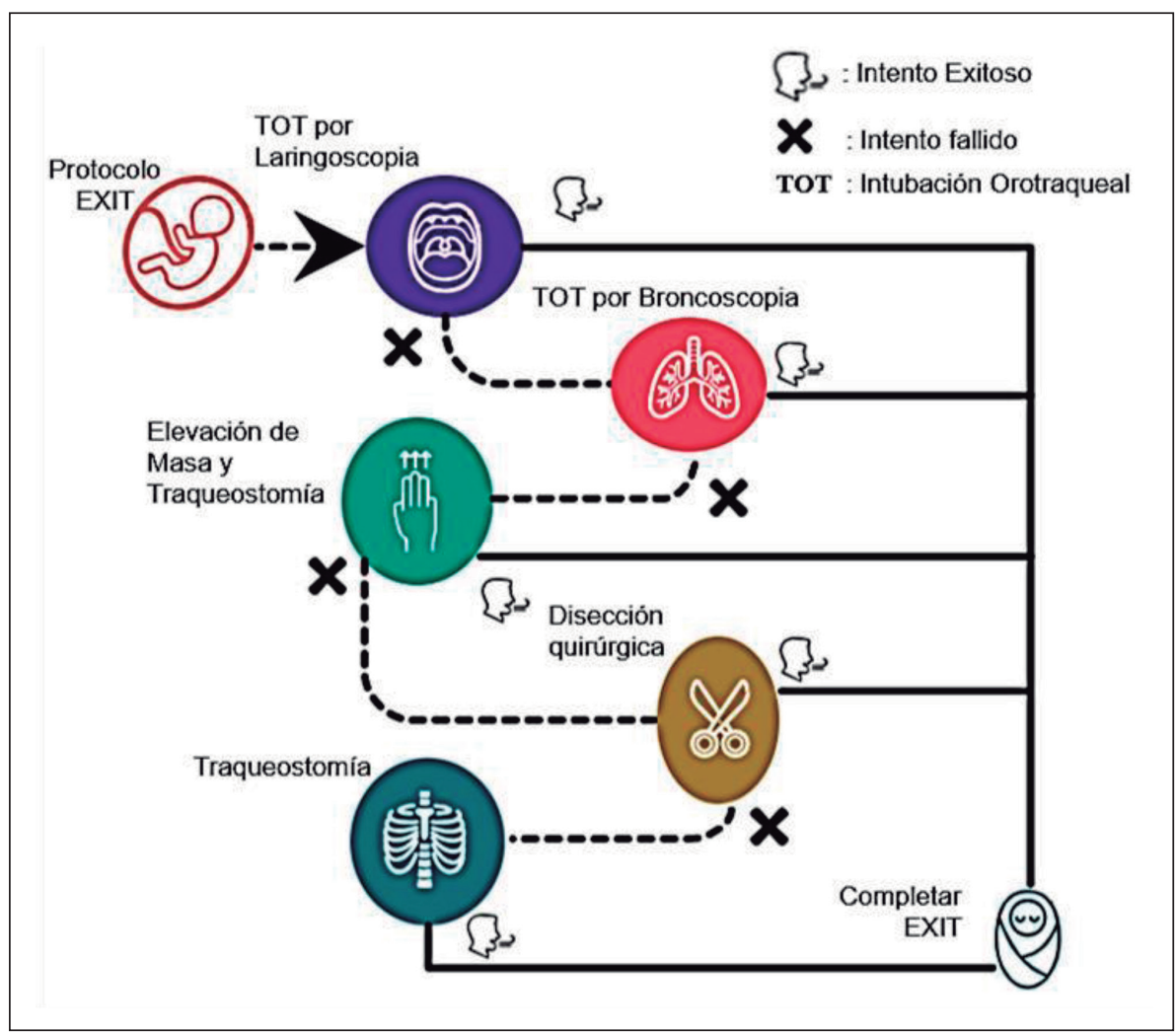
de la técnica EXIT.

a la vía aérea ${ }^{22}$. En los pacientes reportados el neonatólogo logró asegurar la vía aérea con IOT al primer intento.

Cuando se logre asegurar la vía aérea se suspende la anestesia, se pinza y se corta el cordón umbilical y se procede a extraer el resto del cuerpo fetal. Finalmente, se realiza el alumbramiento administrando medicamentos uterotónicos para el cierre de las paredes uterina y abdominal ${ }^{12}$.

Debe tenerse en cuenta que la técnica EXIT es una medida transitoria de asegurar la ventilación del paciente y debe realizarse por tanto el abordaje oportuno de la masa cervical que genera, o que potencialmente puede provocar la obstrucción de vía aérea. La RM es la mejor manera de identificar con mayor claridad el compromiso del paciente al permitir la diferenciación del tejido alterado con respecto al normal ${ }^{23}$. En los casos de este estudio, se realizó RM antenatal y postnatalmente permitiendo al equipo la toma de decisiones dirigidas (dentro de las cuales se incluyó la técnica EXIT). 
Aunque es segura y con alta tasa de éxito, la técnica EXIT puede generar complicaciones maternoperinatales. La madre requiere anestesia general y tiempos intraquirúrgicos prolongados; debido a que la relajación uterina es necesaria para mantener la perfusión placentaria y el intercambio gaseoso, hay mayor riesgo de pérdida de volumen sanguíneo e histerectomía $^{24}$. En el feto, las complicaciones se relacionan con la imposibilidad de conservar el intercambio gaseoso útero-placentario por compresión del cordón umbilical, abrupcio placentario o la relajación uterina ${ }^{17,25}$. Laje et al. reportaron la muerte del $26 \%$ de pacientes con teratoma manejados con la técnica ${ }^{7}$ y Macarthur et al reportaron que la mortalidad relacionada con esta técnica aumenta $36 \%$ si tiene alguna anomalía cromosómica $^{26}$. En los pacientes de este estudio no se presentaron complicaciones.

\section{Conclusiones}

La técnica EXIT es una opción para tomar cuando se tiene en frente el riesgo inminente de asfixia perinatal por compresión de la vía aérea en el feto. Para lograr implementarla se debe contar con un equipo multidisciplinar capacitado y el acceso a herramientas tecnológicas que garanticen la supervivencia del recién nacido, por ende, es una técnica que debe ser protocolizada. El diagnóstico temprano de las situaciones fetales en la que se indica es indispensable para su programación oportuna.

\section{Responsabilidades Éticas}

Protección de personas y animales: Los autores declaran que los procedimientos seguidos se conformaron a las normas éticas del comité de experimentación humana responsable y de acuerdo con la Asociación Médica Mundial y la Declaración de Helsinki.

Confidencialidad de los datos: Los autores declaran que han seguido los protocolos de su centro de trabajo sobre la publicación de datos de pacientes.

Derecho a la privacidad y consentimiento informado: Los autores han obtenido el consentimiento informado de los padres (tutores) de la paciente y/o sujetos referidos en el artículo. Este documento obra en poder del autor de correspondencia

\section{Conflicto de intereses}

Los autores declaran no tener conflicto de intereses.

\section{Referencias}

1. Hamid-Sowinska A, Ropacka-Lesiak M, Breborowicz GH. Congenital high airway obstruction syndrome. Neuro Endocrinol Lett. 2011;32:623-6.

2. Elmasalme F, Giacomantonio M, Clarke KD, Othman E, Matbouli S. Congenital cervical teratoma in neonates. Case report review. Eur J Pediatr Surg. 2000;10:252-7.

3. Howarth ES, Draper ES, Budd JLS, Konje JC, Clarke M, Kurinczuk JJ. Populationbased study of the outcome following the prenatal diagnosis of cystic hygroma. Prenat Diagn. 2005;25:286-91.

4. Gaffuri M, Torretta S, Iofrida E, et al. Multidisciplinary management of congenital giant head and neck masses: Our experience and review of the literature. J Pediatr Surg. 2018:733-9.

5. Norris M, Joseph J, Leighton B. Anesthesia for Perinatal Surgery. Am J Perinatol. 1989;6:39-40.

6. Laje P, Johnson MP, Howell LJ, et al. Ex utero intrapartum treatment in the management of giant cervical teratomas. J Pediatr Surg. 2012;47:1208-16.

7. Laje P, Peranteau WH, Hedrick HL, et al. Ex utero intrapartum treatment
(EXIT) in the management of cervical lymphatic malformation. J Pediatr Surg. 2015;50:311-4.

8. Laje P, Howell LJ, Johnson MP, Hedrick HL, Flake AW, Adzick NS. Perinatal management of congenital oropharyngeal tumors: The ex utero intrapartum treatment (EXIT) approach. J Pediatr Surg. 2013;48:2005-10.

9. Mychaliska GB, Bealer JF, Graf JL, Rosen MA, Adzick NS, Harrison MR. Operating on placental support: the ex utero intrapartum treatment procedure. J Pediatr Surg. 1997;32:227-30; discussion 230-1.

10. Harrison MR, Adzick NS, Flake AW, et al. Correction of congenital diaphragmatic hernia in utero VIII: Response of the hypoplastic lung to tracheal occlusion. J Pediatr Surg. 1996;31:1339-48.

11. Masahata K, Soh H, Tachibana K, et al. Clinical outcomes of ex utero intrapartum treatment for fetal airway obstruction. Pediatr Surg Int. 2019;35:835-43.

12. Cruz-Martínez R. MA. Técnica EXIT (Ex Utero Intrapartum Treatment): indicaciones, limitaciones, riesgos y evolución a la técnica de intubación traqueal fetal endoscópica (FETI) The
EXIT procedure : Indications, limitations, risks and progress to the. Rev Mex Ginecol y Obstet. 2015;83:58-65.

13. Taghavi K, Beasley S. The ex utero intrapartum treatment (EXIT) procedure: Application of a new therapeutic paradigm. J Paediatr Child Health. 2013;49:E420-7.

14. Hedrick MH, Ferro MM, Filly RA, Flake AW, Harrison MR, Scott Adzick $\mathrm{N}$. Congenital high airway obstruction syndrome (CHAOS): A potential for perinatal intervention. J Pediatr Surg. 1994;29:271-4.

15. Hirose S, Harrison MR. The ex utero intrapartum treatment (EXIT) procedure. Semin Neonatol. 2003;8:207-14.

16. Hirose S., Farmer DL., Lee H., et al. The Ex Utero Intrapartum Treatment Procedure: Looking Back at the EXIT. J Pediatr Surg. 2004:39:375-80.

17. Zadra N, Giusti F, Midrio P. Ex utero intrapartum surgery (EXIT): Indications and anaesthetic management. Best Pract Res Clin Anaesthesiol. 2004;18:259-71.

18. Marwan A, Crombleholme TM. The EXIT procedure: principles, pitfalls, and progress. Semin Pediatr Surg. 2006;15:107-15. 
19. Hirose S, Sydorak RM, Tsao K, et al. Spectrum of intrapartum management strategies for giant fetal cervical teratoma. J Pediatr Surg. 2003;38:446-50.

20. Chmait RH, Chon AH, Anselmo D, et al. In utero Fetal Intubation for a Large Neck Mass: A Minimally Invasive EXIT Option. Fetal Diagn Ther. 2019;45:275-80.

21. Prickett K, Javia L. Fetal Evaluation and Airway Management. Clin Perinatol. 2018:609-28.

22. Ryan G, Somme S, Crombleholme TM. Airway compromise in the fetus and neonate: Prenatal assessment and perinatal management. Semin Fetal Neonatal Med. 2016;21:230-9.

23. Kathary N, Bulas DI, Newman KD, Schonberg RL. MRI imaging of fetal neck masses with airway compromise: utility in delivery planning. Pediatr Radiol. 2001;31:727-31.

24. Butwick A, Aleshi P, Yamout I. Obstetric hemorrhage during an exit procedure for severe fetal airway obstruction. Can J Anesth. 2009;56:437-42.

25. Moldenhauer JS. Ex Utero Intrapartum Therapy. Semin Pediatr Surg. 2013;22:449.

26. MacArthur CJ. Prenatal diagnosis of fetal cervicofacial anomalies. Curr Opin Otolaryngol Head Neck Surg. 2012;20:482-90.

27. Agarwal A, Rosenkranz E, Yasin S, Swaminathan S. EXIT procedure for fetal mediastinal teratoma with large pericardial effusion: a case report with review of literature. J Matern Neonatal Med. 2018;31:1099-103.
28. Aubin A, Pondaven S, Bakhos D, Lardy H, Robier A, Lescanne E. Oropharyngeal teratomas in newborns: Management and outcome. Eur Ann Otorhinolaryngol Head Neck Dis. 2014;131:271-5.

29. Matte GS, Connor KR, Toutenel NA, Gottlieb D, Fynn-Thompson F. A Modified EXIT-to-ECMO with Optional Reservoir Circuit for Use during an EXIT Procedure Requiring Thoracic Surgery. J Extra Corpor Technol. 2016;48:35-8.

30. Castillo Salinas F, Elorza Fernández D, Gutiérrez Laso A, et al. Recommendations for respiratory support in the newborn (IV). High frequency ventilation, exutero intrapartum treatment (EXIT), extracorporeal membrane oxygenation (ECMO). An Pediatr. 2017;87:295.e1-295. e7. 